\title{
From CheckMate 227 to CheckMate 9LA: rethinking the status of chemotherapy in the immunotherapy era-chemo-free or chemo-reform?
}

\author{
Haiyi Deng, Chengzhi Zhou
}

State Key Laboratory of Respiratory Disease, National Clinical Research Centre for Respiratory Disease, Guangzhou Institute of Respiratory Health, First Affiliated Hospital, Guangzhou Medical University, Guangzhou, China

Correspondence to: Chengzhi Zhou. Guangzhou Institute of Respiratory Health, State Key Laboratory of Respiratory Disease, First Affiliated Hospital, Guangzhou Medical University, 151 Yanjiang Road, Guangzhou 510120, China. Email: doctorzcz@163.com.

Submitted Mar 06, 2021. Accepted for publication Apr 12, 2021.

doi: $10.21037 /$ tlcr-21-179

View this article at: http://dx.doi.org/10.21037/tlcr-21-179

Non-small cell lung cancer (NSCLC) accounts for $85 \%$ of all primary lung cancers (1). A subset of NSCLC patients with driver mutations is able to benefit from targeted therapy (2); however, platinum-based chemotherapy is still received by patients who lack driver mutations. For patients with NSCLC, immune checkpoint inhibitors (ICIs), including programmed death-1 (PD-1), programmed death-ligand 1 (PD-L1), and cytotoxiclymphocyte antigen 4 (CTL-4) inhibitors, have brought great progress. The KEYNOTE-024 study showed that, as a first-line treatment, pembrolizumab monotherapy remarkably improved the treatment efficacy in patients with advanced NSCLC and PD-L1 expression $\geq 50 \%$ compared with chemotherapy (3). In KEYNOTE 042, compared with chemotherapy, progression-free survival (PFS) of pembrolizumab monotherapy was not statistically different in the PD-L1 expression $\geq 20 \%$ and $\geq 1 \%$ groups, but it was significantly longer in the PD-L1 expression $\geq 50 \%$ group. In addition, overall survival (OS) in the ICI group was significantly longer than in the chemotherapy group in all three populations with different PD-L1 expression, and the difference is largest in the PD-L1 expression $\geq 50 \%$ group [hazard ratio $(\mathrm{HR})=0.69$ vs. 0.77 vs. 0.81] (4). Similarly, IMpower 110 demonstrated atezolizumab alone did not differ in OS compared to chemotherapy in the PD-L1 expression $\geq 1 \%$ group, but significantly prolonged OS in the PD-L1 expression $\geq 50 \%$ group (5). The good efficacy of immunotherapy combined with doublet chemotherapy was confirmed in non-squamous and squamous NSCLC by KEYNOTE-189 (6) and KEYNOTE-407 (7), respectively; however, patients with a high PD-L1 expression level were found to be more likely to respond to ICIs than those with lower PD-L1 expression level. In the IMpower 150 (8) and IMpower 130 (9) studies, the efficacy of atezolizumab combined with chemotherapy with or without bevacizumab was superior to chemotherapy in the PD-L1 high expression group.

In part 1 of the CheckMate 227 phase 3 trial (10), patients with stage IV or recurrent NSCLC were randomly assigned at a ratio of $1: 1: 1$ to receive nivolumab plus ipilimumab, nivolumab alone, or chemotherapy. Among all the trial participants, the median OS was 17.1 months with nivolumab plus ipilimumab versus 13.9 months with chemotherapy. In patients with a PD-L1 expression level of $1 \%$ or more, the median OS was longer with nivolumab plus ipilimumab [17.1 months; 95\% confidence interval (CI), 15.0 to 20.1] than with chemotherapy (14.9 months; 95\% CI, 12.7-16.7), with a HR for death of $0.79(95 \%$ CI, 0.65-0.96). Among the participants with a PD-L1 expression level of less than $1 \%$, the median OS reached 17.2 and 12.2 months in the nivolumab plus ipilimumab group and the chemotherapy group, respectively (HR $=0.62 ; 95 \%$ CI, 0.48-0.78). In CheckMate 227, dual immunotherapy without chemotherapy (chemo-free) was demonstrated to have survival benefit regardless of patients' PD-L1 expression levels, providing an additional option for the front-line therapy of patients with advanced NSCLC, especially those with negative PD-L1 expression. The PFS of patients with a high tumor mutational burden $(\geq 10$ mutations per megabase) in the dual immunotherapy group 
was significantly longer than that in the chemotherapy group, which validated tumor mutational burden as a biomarker for dual immunotherapy (11). Furthermore, patients in the immunotherapy group experienced slightly fewer severe adverse events than those in the chemotherapy group.

Paz-Ares and colleagues recently reported the results of a double-blind phase III study of nivolumab plus ipilimumab in combination with 2 cycles of chemotherapy (CheckMate 9LA) (12). The primary endpoint was OS, and the secondary endpoints included PFS, overall response rate (ORR), and OS, PFS, and ORR by PD-L1 expression level. A total of 719 eligible patients were randomly assigned at a 1:1 ratio to receive nivolumab (360 $\mathrm{mg}$ Q3W) plus ipilimumab (1 mg/kg Q6W) with either 2 cycles of histology-based platinum doublet chemotherapy $(\mathrm{n}=361)$ or 4 cycles of chemotherapy alone ( $n=358$ ). In both treatment groups, the chemotherapy regimens were carboplatin plus paclitaxel for squamous NSCLC and carboplatin/cisplatin plus pemetrexed for non-squamous NSCLC.

CheckMate 9LA (12) reported significant improvement in the primary endpoints in the experimental group compared to the control group: median OS (14.1 vs. 10.7 months; HR, 0.69; 95\% CI, 0.55-0.87, $\mathrm{P}=0.00065)$. With a further 3.5 months of follow-up, the median OS was 15.6 months in the doublet immunotherapy-chemotherapy arm and 10.9 months in the chemotherapy arm. A 12 -month OS benefit was observed in the experimental group when compared to the control group ( $63 \%$ vs. 47\%). The participants in the experimental group experienced improved PFS compared to those in the control group (6.8 vs. 5.0 months). Similarly, a significant improvement was also observed in the ORR with combination therapy $(37.7 \%$ vs. $25.1 \% ; \mathrm{P}=0.00030)$. In the $\mathrm{PD}-\mathrm{L} 1$ expression $\geq 1 \%$ group, the median OS was 15.8 months and 10.9 months in the combination and chemotherapy groups, respectively (HR $=0.64 ; 95 \%$ CI, 0.50-0.82). Among patients with PD-L1 expression $<1 \%$, the median OS reached 16.8 months and 9.8 months with combination therapy and chemotherapy, respectively ( $\mathrm{HR}=0.62 ; 95 \% \mathrm{CI}, 0.45-0.85$ ). Thus, CheckMate 9LA confirmed that the survival benefit of double immunotherapy plus chemotherapy was not related to PD-L1 expression. The robust positive results of the clinical trial led the United States Food and Drug Administration to approve nivolumab combined with ipilimumab and 2 cycles of chemotherapy for the treatment of advanced NSCLC without targetable driver mutations.

Previous studies have shown that the early survival curves of patients who receive immunotherapy are entangled with, or are lower than, those of chemotherapy-treated patients $(4,13,14)$. CheckMate 9LA demonstrated the OS and PFS curves of the combination treatment group were separated from those of the chemotherapy group at an early point without crossover. The addition of limited chemotherapy may benefit those patients who have not benefited from immunotherapy in the first place. Additionally, CheckMate 9LA (HR of 0.66) may have a greater difference in OS between the experimental group and the control group, than did CheckMate 227 (HR of 0.73) (10). The effect of immunotherapy alone may be restricted due to extensive tumor antigen heterogeneity that prevents the immune system from triggering effective attacks (15). Chemotherapy, even at a low dose, has synergistic effects when combined with immunotherapy. Chemotherapy can release antigens, induce immunogenic cell death, activate the antitumor immune response, and increase PD-L1 expression on malignant cells (16-18). Importantly, chemotherapy can convert immunologically "cold" tumors into "hot" tumors with infiltration by dendritic cells and CD8 + cytotoxic T lymphocytes, promoting a response to ICIs in the early stage (19). Additionally, limited chemotherapy may minimize the adverse events associated with fulldose chemotherapy. In CheckMate 9LA, adverse events of grade 3-5 were increased slightly in the experimental group compared to the control group; nevertheless, severe myelosuppression was more common in patients treated with chemotherapy alone.

Two cycles of chemotherapy can enhance the efficacy of immunotherapy without suppressing immunogenicity or increasing the incidence of adverse events. It is not clear whether the addition of other chemotherapeutic modalities to immunotherapy can also attain survival benefits. In vitro experiments confirmed that the immunomodulatory effects of pemetrexed or paclitaxel appeared to be reduced when combined with platinum (20). Whether ICIs can be combined with platinum-free chemotherapy is also worthy of further study. André et al. demonstrated that metronomic chemotherapy combined with ICIs is a promising treatment that can improve the activity of ICIs and maintain the chemotherapeutic effect (21). The addition of chemotherapy to immunotherapy, whether single or dual, should not be restricted to the regimen of 4 courses of standard platinum-containing chemotherapy. Thus, we propose the concept of "chemo-reform": the addition of post-reform chemotherapy to immunotherapy, including single-drug chemotherapy (without platinum), platinum 
alone, low-dose chemotherapy, chemotherapy with the adjusted course, or cycle interval-adjusted chemotherapy.

So is the status of chemotherapy in the era of immunotherapy chemo-free or chemo-reform? CheckMate 227 and CheckMate 9LA showed that chemo-reform may be more effective than chemo-free, with the advantage of early survival benefit. However, the incidence of severe adverse events was higher with chemo-reform than with chemo-free. Therefore, this question remains unanswered and warrants further study.

In summary, both CheckMate 227 and CheckMate 9LA have confirmed that a chemo-free or chemo-reform doublet immunotherapy approach significantly improves patients' OS regardless of their PD-L1 expression status, and has a favorable safety profile. Additionally, CheckMate 9LA showed the addition of 2 chemotherapy cycles in double immunotherapy has avoided the typical early intersection of the curves, saving those patients who do not respond to immunotherapy alone. How chemotherapy and immunotherapy can be combined to achieve the best effect and reduce adverse events is deserving of further study.

\section{Acknowledgments}

Funding: None.

\section{Footnote}

Provenance and Peer Review: This article was a standard submission to the journal. The article has undergone external peer review.

Conflicts of Interest: Both authors have completed the ICMJE uniform disclosure form (available at http://dx.doi. org/10.21037/tlcr-21-179). The authors have no conflicts of interest to declare.

Ethical Statement: The authors are accountable for all aspects of the work in ensuring that questions related to the accuracy or integrity of any part of the work are appropriately investigated and resolved.

Open Access Statement: This is an Open Access article distributed in accordance with the Creative Commons Attribution-NonCommercial-NoDerivs 4.0 International License (CC BY-NC-ND 4.0), which permits the noncommercial replication and distribution of the article with the strict proviso that no changes or edits are made and the original work is properly cited (including links to both the formal publication through the relevant DOI and the license). See: https://creativecommons.org/licenses/by-nc-nd/4.0/.

\section{References}

1. Duma N, Santana-Davila R, Molina JR. Non-Small Cell Lung Cancer: Epidemiology, Screening, Diagnosis, and Treatment. Mayo Clin Proc 2019;94:1623-40.

2. Giustini NP, Jeong AR, Buturla J, et al. Advances in Treatment of Locally Advanced or Metastatic Non-Small Cell Lung Cancer: Targeted Therapy. Clin Chest Med 2020;41:223-35.

3. Reck M, Rodríguez-Abreu D, Robinson AG, et al. Pembrolizumab versus Chemotherapy for PD-L1Positive Non-Small-Cell Lung Cancer. N Engl J Med 2016;375:1823-33.

4. Mok TSK, Wu YL, Kudaba I, et al. Pembrolizumab versus chemotherapy for previously untreated, PD-L1expressing, locally advanced or metastatic non-small-cell lung cancer (KEYNOTE-042): a randomised, open-label, controlled, phase 3 trial. Lancet 2019;393:1819-30.

5. Herbst RS, Giaccone G, de Marinis F, et al. Atezolizumab for First-Line Treatment of PD-L1-Selected Patients with NSCLC. N Engl J Med 2020;383:1328-39.

6. Gandhi L, Rodríguez-Abreu D, Gadgeel S, et al. Pembrolizumab plus Chemotherapy in Metastatic NonSmall-Cell Lung Cancer. N Engl J Med 2018;378:2078-92.

7. Paz-Ares L, Luft A, Vicente D, et al. Pembrolizumab plus Chemotherapy for Squamous Non-Small-Cell Lung Cancer. N Engl J Med 2018;379:2040-51.

8. Socinski MA, Jotte RM, Cappuzzo F, et al. Atezolizumab for First-Line Treatment of Metastatic Nonsquamous NSCLC. N Engl J Med 2018;378:2288-301.

9. West H, McCleod M, Hussein M, et al. Atezolizumab in combination with carboplatin plus nab-paclitaxel chemotherapy compared with chemotherapy alone as firstline treatment for metastatic non-squamous non-smallcell lung cancer (IMpower130): a multicentre, randomised, open-label, phase 3 trial. Lancet Oncol 2019;20:924-37.

10. Hellmann MD, Paz-Ares L, Bernabe Caro R, et al. Nivolumab plus Ipilimumab in Advanced Non-Small-Cell Lung Cancer. N Engl J Med 2019;381:2020-31.

11. Hellmann MD, Ciuleanu TE, Pluzanski A, et al. Nivolumab plus Ipilimumab in Lung Cancer with a High Tumor Mutational Burden. N Engl J Med 2018;378:2093-104.

12. Paz-Ares L, Ciuleanu TE, Cobo M, et al. First-line 
nivolumab plus ipilimumab combined with two cycles of chemotherapy in patients with non-small-cell lung cancer (CheckMate 9LA): an international, randomised, open-label, phase 3 trial. Lancet Oncol 2021;22:198-211. Erratum in: Lancet Oncol 2021;22:e92.

13. Rittmeyer A, Barlesi F, Waterkamp D, et al. Atezolizumab versus docetaxel in patients with previously treated non-small-cell lung cancer (OAK): a phase 3, openlabel, multicentre randomised controlled trial. Lancet 2017;389:255-65.

14. Borghaei H, Paz-Ares L, Horn L, et al. Nivolumab versus Docetaxel in Advanced Nonsquamous Non-Small-Cell Lung Cancer. N Engl J Med 2015;373:1627-39.

15. Leonetti A, Wever B, Mazzaschi G, et al. Molecular basis and rationale for combining immune checkpoint inhibitors with chemotherapy in non-small cell lung cancer. Drug Resist Updat 2019;46:100644.

16. Wang Q, Ju X, Wang J, et al. Immunogenic cell death in anticancer chemotherapy and its impact on clinical studies.
Cancer Lett 2018;438:17-23.

17. Kyi C, Postow MA. Immune checkpoint inhibitor combinations in solid tumors: opportunities and challenges. Immunotherapy 2016;8:821-37.

18. Zhang P, Ma Y, Lv C, et al. Upregulation of programmed cell death ligand 1 promotes resistance response in nonsmall-cell lung cancer patients treated with neo-adjuvant chemotherapy. Cancer Sci 2016;107:1563-71.

19. Galluzzi L, Humeau J, Buqué A, et al. Immunostimulation with chemotherapy in the era of immune checkpoint inhibitors. Nat Rev Clin Oncol 2020;17:725-41.

20. Schaer DA, Geeganage S, Amaladas N, et al. The Folate Pathway Inhibitor Pemetrexed Pleiotropically Enhances Effects of Cancer Immunotherapy. Clin Cancer Res 2019;25:7175-88.

21. André N, Carré M, Pasquier E. Metronomics: towards personalized chemotherapy? Nat Rev Clin Oncol 2014;11:413-31.

Cite this article as: Deng H, Zhou C. From CheckMate 227 to CheckMate 9LA: rethinking the status of chemotherapy in the immunotherapy era-chemo-free or chemo-reform? Transl Lung Cancer Res 2021;10(4):1924-1927. doi: 10.21037/ tlcr-21-179 